06

\title{
Уравнение состояния и поверхностные свойства аморфного железа
}

\author{
(c) М.Н. Магомедов
}

Институт проблем геотермии и возобновляемой энергетики фрилиал Объединенного института высоких температур РАН, 367030 Махачкала, Россия

e-mail: mahmag4@mail.ru

Поступило в Редакцию 24 февраля 2020 г.

В окончательной редакции 27 марта 2020 г.

Принято к публикации 20 апреля 2020 г.

Показано, что на нелинейной зависимости первого координационного числа $\left(k_{n}\right)$ от коэффициента упаковки $\left(k_{p}\right)$ структуры однокомпонентного вещества можно выделить три особые точки, соответствующие аморфным структурам. На основе парного межатомного потенциала Ми-Леннард-Джонса рассчитаны уравнения состояния и свойства железа как для этих трех аморфных структур, так и для кристаллического состояния. Показано, что при $k_{p}=0.45556$ и $k_{n}=6.2793$ достигается минимум химического потенциала, т.е. эта упаковка является термодинамически устойчивой аморфной структурой, соответствующей жидкой фазе. Энергетически эквивалентная ей точка с таким же значением $k_{n}$, но с $k_{p}=0.6237$, является термодинамически неустойчивой аморфной структурой, соответствующей твердой фазе. Показано, что удельная поверхностная энергия аморфного твердого металла больше, чем аморфной жидкой фазы, но меньше, чем металла в кристаллическом состоянии. Это должно приводить к тому, что поверхность кристаллического металла должна стремиться к аморфизации.

Ключевые слова: структура, аморфизация, температура Дебая, железо, уравнение состояния, модуль упругости, поверхностная энергия.

DOI: $10.21883 /$ JTF.2020.10.49806.62-20

\section{Введение}

Известно, что при низкотемпературной конденсации или при скоростном охлаждении расплава (т.е. при закалке) многие простые (т.е. однокомпонентные) вещества переходят в аморфное состояние $[1,2]$. Свойства аморфного вещества существенно отличаются от свойств его кристаллического состояния. Но структура аморфного состояния точно не определена, что приводит к широкому интервалу значений как первого координационного числа $\left(k_{n}\right)$, так и коэффициента упаковки $\left(k_{p}\right)$, которые характеризуют эту аморфную структуру [3-8]. В большинстве экспериментальных и теоретических исследований утверждается, что аморфному состоянию простого вещества соответствует область: $0.55 \leq k_{p} \leq 0.64[1,4,7,8]$.

С другой стороны, для определения аморфной структуры в [5] была использована функция $k_{n}\left(k_{p}\right)$, полученная на основании изучения зависимости $k_{n}\left(k_{p}\right)$ для 4-х кубических структур: гранецентрированной кубической (ГЦК: $\left.k_{n}=12 ; \quad k_{p}=\pi 2^{1 / 2} / 6=0.7405\right)$, объемно центрированной кубической (ОЦК: $k_{n}=8$; $\left.k_{p}=\pi 3^{1 / 2} / 8=0.6802\right)$, простой кубической упаковки (ПКУ: $k_{n}=6 ; k_{p}=\pi / 6=0.5236$ ) и алмазной кубической упаковки (АКУ: $k_{n}=4 ; k_{p}=\pi 3^{1 / 2} / 16=0.3401$ ). Зависимость $k_{n}\left(k_{p}\right)$ с коэффициентом достоверности, равным единице, описывается полиномом 3-й степени следующего вида: ${ }^{1}$

$$
\begin{aligned}
k_{n}\left(k_{p}\right)= & -71.76782+467.78914 \cdot k_{p} \\
& -925.48451 \cdot k_{p}^{2}+603.01146 \cdot k_{p}^{3} .
\end{aligned}
$$

Другими авторами для зависимости $k_{n}\left(k_{p}\right)$ в аморфных структурах были предложены следующие выражения:

$$
\begin{gathered}
k_{n}\left(k_{p}\right)=1.275+6.1383 \cdot k_{p}+5.5116 \cdot k_{p}^{2}[6], \\
k_{n}\left(k_{p}\right)=\alpha_{H} 2(3)^{1 / 2} k_{p} /\left(1-k_{p}\right)[7,8],
\end{gathered}
$$

где корректирующий множитель брали в виде: $\alpha_{H}=1$ в [7], и $\alpha_{H}=1.1$ в [8].

На рис. 1 показаны графики этих зависимостей: 1 зависимость (1) из [5], 2 - зависимость (2) из [6], 3 - зависимость (3) при $\alpha_{H}=1$ из [7], линия 4 зависимость (3) при $\alpha_{H}=1.1$ из [8]. Из рис. 1 видно, что зависимость (1) в отличие от зависимостей из [68] лучше описывает весь диапазон возможных структур однокомпонентных веществ.

Как было показано в [5], и как это видно из рис. 1, зависимость (1) имеет $\mathrm{N}$-петлю, и в области $5.855 \leq k_{n} \leq 6.2793$ и $0.4 \leq k_{p} \leq 0.6237$ одному значению $k_{n}$ соответствуют два или три значения $k_{p}$. Поэтому

\footnotetext{
1 Здесь и далее аппроксимация соответствующих зависимостей полиномами различных степеней осуществлялась методом наименьших квадратов, встроенным в графопостроитель Origin.
} 


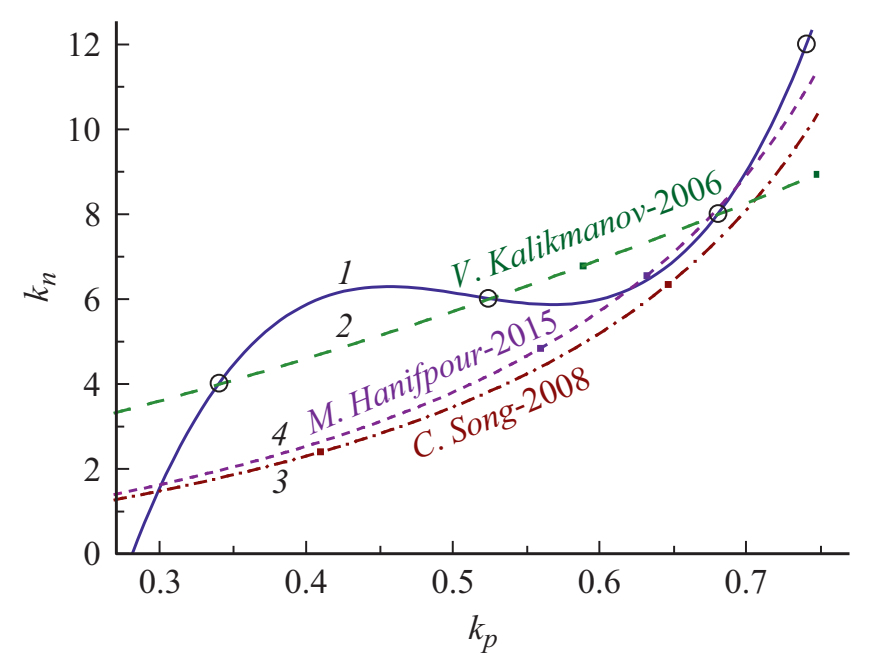

Рис. 1. Зависимость первого координационного числа от коэффициента упаковки простого вещества. Символы данные для 4-х кубических структур: ГЦК, ОЦК, ПКУ и АКУ. Сплошная линия 1 - зависимость (1) из [5], штриховая линия 2 - зависимость (2) из [6], пунктирная линия 3 - зависимость (3) при $\alpha_{H}=1$ из [7], штрихпунктирная линия $4-$ зависимость (3) при $\alpha_{H}=1.1$ из [8].

эта структурная область была определена в [5], как область „случайной упаковки“. Это согласуется с оценками координационного числа в „случайной упаковке“, полученными другими авторами в работах $[1,3,6-8]$ : $k_{n}=6-6.08$.

Как было указано в [9], на зависимости (1) можно выделить три характерные точки: две точки экстремумов зависимости $k_{n}\left(k_{p}\right)$ и точку пересечения зависимости $k_{n}\left(k_{p}\right)$ с касательной в точке максимума данной зависимости:

1 - рыхлая аморфная упаковка:

$$
k_{p}(1)=0.4555, \quad k_{n}(1)=6.2793,
$$

2 - средняя аморфная упаковка:

$$
k_{p}(2)=0.56762, \quad k_{n}(2) 5.855,
$$

3 - плотная аморфная упаковка:

$$
k_{p}(3)=0.623, \quad k_{n}(3)=6.2793 .
$$

Отметим, что значения $k_{p}$ в экстремумах, т.е. $k_{p}$ (1) и $k_{p}$ (2) близки к оценкам, полученным моделированием системы жестких сфер методом Монте-Карло в работе [10]: жидкость и кристалл сосуществуют между точкой замерзания: $k_{\text {psolidus }}=0.494 \pm 0.002$, и точкой плавления: $k_{\text {pliquidus }}=0.545 \pm 0.002$.

В [9] было показано, что в точке 1 достигается минимум удельной (на атом) свободной энергия Гельмгольца $\left(f_{H}\right)$, т.е. эта упаковка является термодинамически устойчивой структурой. Так же было показано, что в точках 1 и 3 значения $f_{H}$ совпадают: $f_{H}(1)=f_{H}(3)$.
В [9] был проведен расчет температуры Дебая $(\Theta)$ в точках 1 и 2 для 12 однокомпонентных металлов, а также для $\mathrm{Si}$ и $\mathrm{Ge}$. Результаты расчета показали хорошее согласие с экспериментальными оценками величины $\Theta$ для аморфного состояния. Но величина $\Theta$ определяется в основном значением $k_{n}$, которое в точках 1 и 3 одинаково. Кроме того, расчеты в [9] были проведены при изохорическом изменении аргумента $k_{p}$, т.е. без изучения давления в системе. Также в [9] не было учтено изменение межатомного взаимодействия при вариации структуры вещества. Поэтому в настоящей работе было рассчитано уравнение состояния $P(V, T)$ и свойства аморфного железа в точках 1-3 зависимости (4), с учетом зависимости парного межатомного взаимодействия от структуры железа. Было сделано сравнение полученных в точках 1-3 результатов со свойствами железа в кристаллическом состоянии при $P=0$. На основании полученных результатов был сделан вывод о соответствии указанных в (4) точек параметрам твердого аморфного железа.

\section{1. Метод расчета свойств твердого металла}

Положим, что атомы в металле взаимодействуют посредством парного потенциала Ми-Леннард-Джонса следующего вида [11]:

$$
\varphi(r)=\frac{D}{(b-a)}\left[a\left(\frac{r_{o}}{r}\right)^{b}-b\left(\frac{r_{o}}{r}\right)^{a}\right],
$$

где $D$ и $r_{o}$ - глубина и координата минимума потенциала, $b>a>1$ - параметры.

Тогда, используя приближение „взаимодействия только ближайших соседей“, температуру Дебая как функцию первого координационного числа $\left(k_{n}\right)$ и расстояния между центрами ближайших атомов $\left(c=\left(6 k_{p} v / \pi\right)^{1 / 3}\right)$ можно определить в виде $[11,12]$

$$
\begin{aligned}
& \Theta\left(k_{n}, c\right)=A_{w}\left(k_{n}, c\right) \xi \\
& \times\left[-1+\left(1+\frac{8 D}{k_{\mathrm{B}} A_{w}\left(k_{n}, c\right) \xi^{2}}\right)^{1 / 2}\right] .
\end{aligned}
$$

Здесь $k_{\mathrm{B}}$ - постоянная Больцмана, функция $A_{w}$ определяет энергию „нулевых колебаний“ атомов в кристалле, $v=V / N$ - удельный объем, $V$ и $N-$ объем и число атомов в кристалле:

$$
\begin{gathered}
A_{w}\left(k_{n}, c\right)=K_{R} \frac{5 k_{n} a b(b+1)}{144(b-a)}\left(\frac{r_{o}}{c}\right)^{b+2}, \\
K_{R}=\frac{\hbar^{2}}{k_{\mathrm{B}} r_{o}^{2} m}, \quad \xi=\frac{9}{k_{n}},
\end{gathered}
$$

где $m$ - масса атома, $\hbar-$ постоянная Планка.

Используя приближение „взаимодействия только ближайших соседей“ и описывая колебательный спектр 
вещества по модели Эйнштейна, для удельной (на атом) свободной энергии Гельмгольца можно использовать выражение [11,13]:

$$
\begin{aligned}
& f_{H}\left(k_{n}, c, T\right)=\left(\frac{k_{n}}{2}\right) D \cdot U(R)+3 k_{\mathrm{F}} \Theta_{E}\left(k_{n}, c\right) \\
& \times\left\{\left(\frac{1}{2}\right)+\left(\frac{T}{\Theta_{E}\left(k_{n}, c\right)}\right) \ln \left[1-\exp \left(-\frac{\Theta_{E}\left(k_{n}, c\right)}{T}\right)\right]\right\},
\end{aligned}
$$

где $T-$ температура, $\Theta_{E}-$ температура Эйнштейна, которая связана с температурой Дебая соотношением [13]: $\Theta=(4 / 3) \Theta_{E}, R=r_{o} / c-$ относительная линейная плотность вещества, функция потенциальной энергии в соответствии с (5) равна:

$$
U(R)=\frac{a R^{b}-b R^{a}}{b-a}
$$

Исходя из формулы (7), для уравнения состояния и изотермического модуля упругости $\left(B_{T}\right)$ можно получить выражения [14]

$$
\begin{aligned}
P= & -\left(\frac{\partial f_{H}}{\partial v}\right)_{T} \\
= & {\left[\frac{k_{n}}{6} D \cdot U^{\prime} /(R)+3 k_{\mathrm{B}} \Theta_{E} \cdot \gamma \cdot E_{W}\left(\frac{\Theta_{E}}{T}\right)\right] \frac{1}{v}, } \\
B_{T}= & -v\left(\frac{\partial P}{\partial v}\right)_{T}=P+\left[\frac{k_{n}}{18} D \cdot U^{\prime \prime}(R)+3 k_{\mathrm{B}} \Theta_{E} \cdot \gamma \cdot(\gamma-q)\right. \\
& \left.\times E_{w}\left(\frac{\Theta_{E}}{T}\right)-3 N \cdot k_{\mathrm{B}} \cdot \gamma^{2} \cdot T \cdot F_{E}\left(\frac{\Theta_{E}}{T}\right)\right] \frac{1}{v}
\end{aligned}
$$

Здесь введены следующие функции:

$$
\begin{gathered}
E_{w}(y)=0.5+\frac{1}{[\exp (y)-1]}, \quad F_{E}(y)=\frac{y^{2} \exp (y)}{[\exp (y)-1]^{2}} \\
v=\frac{\pi c^{3}}{6 k_{p}}, \quad U^{\prime}(R)=R\left[\frac{\partial U(R)}{\partial R}\right]=\frac{a b\left(R^{b}-R^{a}\right)}{b-a} \\
U^{\prime \prime}(R)=R\left[\frac{\partial U^{\prime}(R)}{\partial R}\right]=\frac{a b\left(b R^{b}-a R^{a}\right)}{b-a} .
\end{gathered}
$$

Из формулы (6) легко найти выражения для первого $(\gamma)$ и второго $(q)$ параметров Грюнайзена, которые входят в (8) и (9). Они имеют вид

$$
\begin{gathered}
\gamma=-\left(\frac{\partial \ln \Theta}{\partial \ln v}\right)_{T}=\frac{b+2}{6+\left(1+X_{w}\right)}, \\
q=\left(\frac{\partial \ln \gamma}{\partial \ln v}\right)_{T}=\gamma \frac{X_{w}\left(1+2 X_{w}\right)}{1+X_{w}},
\end{gathered}
$$

где введена функция $X_{w}=A_{w} \xi / \Theta$, которая определяет роль квантовых эффектов.
Так как температура Дебая (6) не зависит от температуры при изохорическом нагреве, то изохорную и изобарную теплоемкости можно определить в виде [13]

$$
C_{v}=3 N \cdot k_{\mathrm{B}} \cdot F_{E}\left(\frac{\Theta_{E}}{T}\right), \quad C_{p}=C_{v}\left(1+\gamma \alpha_{p} T\right),
$$

где изобарный коэффициент теплового объемного расширения можно рассчитать по уравнению Грюнайзена [13] следующего вида:

$$
\begin{gathered}
\alpha_{p}=\frac{\gamma C_{v}}{V \cdot B_{T}}=\frac{\gamma \cdot C_{v}}{N \cdot B_{T}\left[\pi r_{o}^{3} /\left(6 k_{p}\right)\right]}\left(\frac{v_{0}}{v}\right), \\
v_{0}=\frac{\pi r_{o}^{3}}{6 k_{p}} .
\end{gathered}
$$

Из выражений (6)-(8) можно получить формулы для химического потенциала (т.е. $g$ - удельной энергии Гиббса) и удельной (на атом) энтропии системы:

$$
\begin{gathered}
g=f_{H}+P \cdot v \\
s=-\left(\frac{\partial f_{H}}{\partial T}\right)_{v}=3 k_{\mathrm{B}}\left\{-\ln \left[1-\exp \left(\frac{\Theta_{E}}{T}\right)\right]\right. \\
\left.+\left(\frac{\Theta_{E}}{T}\right) /\left[\exp \left(\frac{\Theta_{E}}{T}\right)-1\right]\right\} .
\end{gathered}
$$

Для удельной (на единицу площади) поверхностной энергии $(\sigma)$ простого вещества, было получено следующее выражение $[11,15]$ :

$$
\sigma(R, T)=-\frac{k_{n} D R^{2}}{12 \alpha^{2 / 3} r_{o}^{2}} L_{E}(R, T)
$$

где введенные функции имеют следующий вид:

$$
\begin{gathered}
L_{E}(R, T)=U(R)+3 H_{w}(R, T), \\
H_{w}(R, T)=\frac{6 \gamma}{(b+2)} \frac{k_{\mathrm{B}} \Theta_{E}}{D k_{n}} E_{w}\left(\frac{\Theta_{E}}{T}\right) .
\end{gathered}
$$

Как было показано в работах $[11,12,14]$, формулы (6)-(15) справедливы для любых $R-T$-условий, которые соответствуют твердой фазе простого вещества.

Зависимость коэффициента Пуассона от $R-T$ аргументов можно рассчитать по формуле, полученной в работе [16]:

$$
\mu_{p}(R, T)=\frac{1}{2}-\frac{1}{48 X_{s c}(R, T) \cdot \gamma^{2}},
$$

где введено соотношение между поверхностными и объемными силами в виде

$$
X_{s c}(R, T)=\frac{\sigma(R, T)}{c \cdot B_{T}(R, T)} .
$$

Если известны параметры межатомного потенциала (5), то с помощью формализма из (6)-(16) можно рассчитать как уравнение состояния, так и указанные 
свойства однокомпонентного вещества с данной структурой (т.е. при данных значениях $k_{n}$ и $k_{p}$ ) и при любых (соответствующих твердой фазе) значениях удельного объема $v$ и температуры $T$. Так как формализм из (6)-(16) получен при использовании приближения „взаимодействия только ближайших соседей“, и при использовании колебательного спектра модели Эйнштейна, то приложение этого формализма к аморфной структуре не потребует каких-либо доработок.

Отметим, что формализм из (6)-(16) не учитывает наличие электронной подсистемы. Это обосновывается тем, что потенциал (5) описывает парное взаимодействие электронейтральных атомов. Как было показано в $[14,17,18]$, погрешности, возникающие при расчете решеточных свойств металла при исключении из рассмотрения электронной подсистемы, пренебрежимо малы.

\section{2. Межатомное взаимодействие в аморфном металле}

Параметры парного потенциала межатомного взаимодействия (5) определяют из экспериментальных данных вещества с конкретной кристаллической структурой. Но в настоящей работе изучается твердое железо $(m(\mathrm{Fe})=55.847 \mathrm{a} . \mathrm{m} . \mathrm{u})$ с различной структурой в точках 1-3 из (4). Как было показано в [17] параметры межатомного потенциала железа при переходе из ОЦК в ГЦК структуру изменяются. Например, для фазы $\alpha$-Fе, имеющей ОЦК структуру $\left(k_{n}=8, k_{p}=0.6802\right)$, параметры потенциала (5) равны [14]:

$$
\begin{gathered}
r_{o}(8)=2.4775 \cdot 10^{-10} \mathrm{~m}, \quad D(8) / k_{\mathrm{B}}=12561.53 \mathrm{~K}, \\
b(8)=8.37, \quad a(8)=3.09 .
\end{gathered}
$$

Для ГЦК фазы $\gamma$-Fe $\left(k_{n}=12, k_{p}=0.7405\right)$ в [17] были изучены различные наборы параметров потенциала (5). Лучшие результаты расчета свойств были получены при следующих значениях параметров:

$$
\begin{gathered}
r_{o}(12)=2.5445 \cdot 10^{-10} m, \quad D(12) / k_{\mathrm{B}}=8384.467 \mathrm{~K}, \\
b(12)=8.26, \quad a(12)=2.12 .
\end{gathered}
$$

Эти изменения межатомного потенциала происходят из-за перераспределения электронной плотности по парным межатомным связям при изменении структуры кристалла. Поэтому для изучения свойств в точках $1-3$ из (4) необходимо определить зависимость параметров потенциала (5) от параметров структуры простого вещества.

Для определения зависимости $r_{o}\left(k_{p}\right)$ было использовано эмпирическое соотношение между межатомным расстоянием и коэффициентом упаковки кристалла с кубической структурой [19]: если при полиморфном превращении величина коэффициента упаковки $k_{p}$ возрастает в последовательности:

$$
\begin{aligned}
k_{p} & =0.3401(\text { АКУ }) \rightarrow 0.5236(\text { ПКУ }) \\
& \rightarrow 0.6802(\text { ОЦК }) \rightarrow 0.7405(\text { ГЦК },
\end{aligned}
$$

Таблица 1. Значения параметров межатомного потенциала (5) для железа в различных структурных точках

\begin{tabular}{c|c|c|c}
\hline Структура & $\begin{array}{c}D / k_{\mathrm{B}}, \\
\mathrm{K}\end{array}$ & $\begin{array}{c}r_{o}, \\
10^{-10} \mathrm{~m}\end{array}$ & $\begin{array}{c}V_{0}, \\
\mathrm{~cm}^{3} / \mathrm{mol}\end{array}$ \\
\hline $1: k_{p}=0.45556, k_{n}=6.2793$ & 12962.34 & 2.4125 & 9.718 \\
\hline $2: k_{p}=0.56762, k_{n}=5.8550$ & 13061.17 & 2.4386 & 8.056 \\
\hline $3: k_{p}=0.62370, k_{n}=6.2793$ & 12962.34 & 2.4493 & 7.428 \\
\hline ОЦК: $k_{p}=0.6802, k_{n}=8$ & 12561.53 & 2.4775 & 7.049 \\
\hline ГЦК: $k_{p}=0.7405, k_{n}=12$ & 11629.80 & 2.5448 & 7.018
\end{tabular}

то длина межатомной связи возрастает в последовательности:

$$
r_{o}\left(k_{p}\right) / r_{o}(4)=1.02 \rightarrow 1.09 \rightarrow 1.11 \rightarrow 1.14 .
$$

Эту зависимость можно аппроксимировать с коэффициентом достоверности равным единице полиномом 3-й степени следующего вида:

$$
\begin{gathered}
r_{o}\left(k_{p}\right) / r_{o}(8)=0.014478+5.146225 k_{p} \\
-9.186595 k_{p}^{2}+5.514874 k_{p}^{3} .
\end{gathered}
$$

Для определения зависимости глубины потенциала $D$ от параметров структуры были использованы два значения: для ОЦК кристалла и для молекулы $\mathrm{Fe}_{2}$ :

$$
D(8) / k_{\mathrm{B}}=12561.53 \mathrm{~K} \quad \text { и } \quad D(1) / k_{\mathrm{B}}=14192.06 \mathrm{~K} .
$$

Здесь первое значение взято из работы [14], а вторая величина определена из энергии диссоциации молекулы $\mathrm{Fe}_{2}$ из $[19,20]: D(1)=118 \mathrm{~kJ} / \mathrm{mol}$. По этим двум точкам была построена линейная зависимость вида

$$
D\left(k_{n}\right)=D(8)+\{[D(1)-D(8)] / 7\}\left(8-k_{n}\right) .
$$

Таким образом, для железа было получено

$$
D\left(k_{n}\right) / k_{\mathrm{B}}=12561.53+232.933\left(8-k_{n}\right)[\mathrm{K}] .
$$

Ввиду отсутствия каких-либо данных по зависимости степенных параметров потенциала (5) от параметров структуры кристалла простого вещества для области $0.45<k_{p}<0.68$, при расчетах свойств в точках $1-3$ из (4) были взяты данные, соответствующие ОЦК-железу:

$$
b=8.37 \quad \text { и } \quad a=3.09 .
$$

В табл. 1 показаны значения параметров потенциала (5), полученные по формулам (17) и (18) для 5 структур Fe: трех аморфных в точках из (4), ОЦК и ГЦК.

На рис. 2 показано изохорно-изотермическое изменение удельной свободной энергии Гельмгольца $\left(f_{H} / k_{\mathrm{B}}\right.$ в $\left.\left[10^{3} \mathrm{~K}\right]\right)$ с изменением $k_{p}$, полученные для железа, 
Таблица 2. Рассчитанные свойства железа с различной структурой при $300 \mathrm{~K}$ и $P=0$

\begin{tabular}{c|c|c|c|c|c|c|c|c|c|c}
\hline $\begin{array}{c}\text { Струк- } \\
\text { тура }\end{array}$ & $\begin{array}{c}V, \\
\mathrm{~cm}^{3} / \mathrm{mol}\end{array}$ & $v / v_{0}$ & $\begin{array}{c}-g / k_{\mathrm{B}}, \\
\mathrm{K}\end{array}$ & $\begin{array}{c}\Theta, \\
\mathrm{K}\end{array}$ & $\begin{array}{c}B_{T}, \\
\mathrm{GPa}\end{array}$ & $\begin{array}{c}\alpha_{p}, \\
10^{-6} \mathrm{~K}^{-1}\end{array}$ & $C_{p} / N k_{\mathrm{B}}$ & $\begin{array}{c}\sigma(100), \\
10^{-3} \mathrm{~J} / \mathrm{m}^{2}\end{array}$ & $\mu_{p}$ & $s / k_{\mathrm{B}}$ \\
\hline 1 & 9.862 & 1.0148 & 40698 & 381.7 & 93.51 & 43.13 & 2.844 & 1434.63 & 0.389 & 3.252 \\
\hline 2 & 8.182 & 1.0156 & 38270 & 365.4 & 105.58 & 46.33 & 2.867 & 1525.54 & 0.380 & 3.373 \\
\hline 3 & 7.537 & 1.0147 & 40698 & 376.0 & 122.40 & 43.21 & 2.851 & 1716.30 & 0.376 & 3.294 \\
\hline ОЦК & 7.134 & 1.0121 & 50167 & 415.1 & 161.24 & 34.13 & 2.793 & 2202.88 & 0.372 & 3.020
\end{tabular}

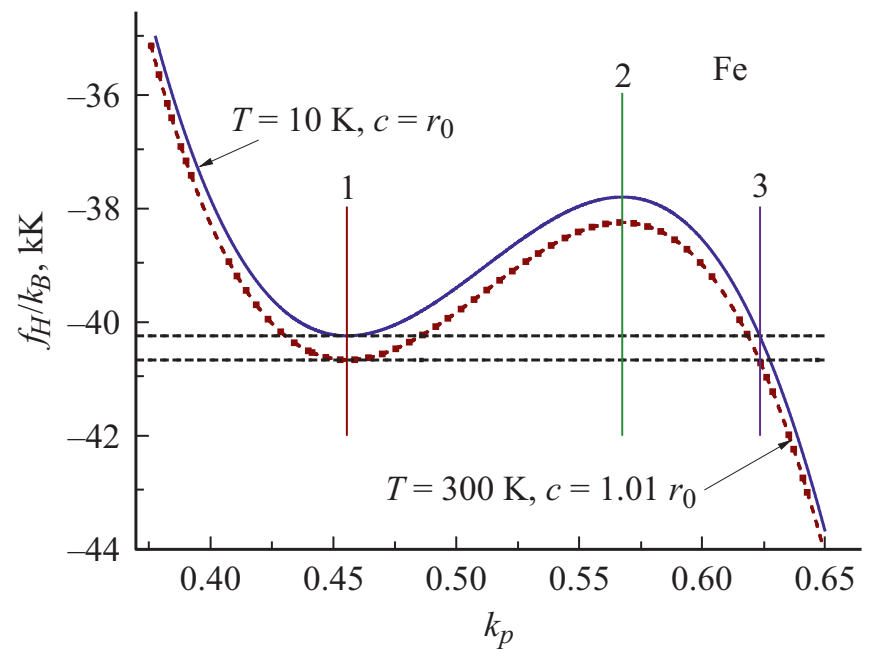

Рис. 2. Изохорно-изотермическая зависимость удельной свободной энергии Гельмгольца $\left(f_{H} / k_{\mathrm{B}}\right.$ в $\left.\left[10^{3} \mathrm{~K}\right]\right)$ от величины $k_{p}$. Сплошная верхняя кривая получена при: $c=r_{0}$ и $T=10 \mathrm{~K}$, штриховая нижняя кривая получена при: $c=1.01 r_{0}$ и $T=300 \mathrm{~K}$. Цифрами $1-3$ и вертикальными линиями указаны положения точек из (4). Горизонтальными штриховыми прямыми показаны касательные в точках 1 .

с учетом изменения параметров потенциала (5) по формулам (6), (7), (17) и (18). Сплошная верхняя кривая получена при $T=10 \mathrm{~K}^{2}$ и при расстоянии между центрами ближайших атомов равном: $c=r_{o}$. Штриховая нижняя кривая получена при $T=300 \mathrm{~K}$ и $c=1.01 r_{o}$. Цифрами 1-3 и вертикальными линиями указаны положения точек 1-3 из (4). Горизонтальными штриховыми прямыми показаны касательные в точках 1 .

Из рис. 2 видно, что изохорно-изотермическая зависимость $f_{H}\left(k_{p}\right)$ при любых температурах образует $\mathrm{S}$-петлю и имеет минимум в точке 1. Это указывает на то, что такая упаковка атомов в точке 1 является термодинамически устойчивой структурой. Также видно, что точки 1 и 3 имеют равную удельную свободную энергию Гельмгольца, которая при $P=0$ совпадает с удельным термодинамическим потенциалом Гиббса, т. е.

\footnotetext{
2 Значение $T=10 \mathrm{~K}$ взято, чтобы показать - как меняется $S$-петля на зависимости $f_{H}\left(k_{p}\right)$ при разных температурах. Жидкая фаза при $T=10 \mathrm{~K}$ находиться в равновесии с твердой (аморфной либо кристаллической) фазой не может.
}

с химическим потенциалом. Поэтому свойства железа были изучены для этих двух аморфных структур в точках 1 и 3 из (4). Для сравнения были также рассчитаны свойства ОЦК-железа.

\section{3. Результаты расчета свойств Fe для различных структур}

Определив зависимость параметров потенциала (5) от структуры железа и используя формализм (6)-(16), были рассчитаны термодинамические свойства статической упаковки железа в точках 1, 3 и для ОЦК кристалла. На основе полученных результатов было показано, что структуру в точке 3 можно считать соответствующей твердому аморфному состоянию железа. Статическая упаковка - это структура, в которой отсутствует самодиффузия атомов по объему системы.

В табл. 2 представлены значения рассчитанных свойств железа для структур 1-3 из (4) и для ОЦКFe. Расчеты сделаны при $T=300 \mathrm{~K}$ и $P=0$. Экспериментальные значения свойств ОЦК-Fе при $T=300 \mathrm{~K}$

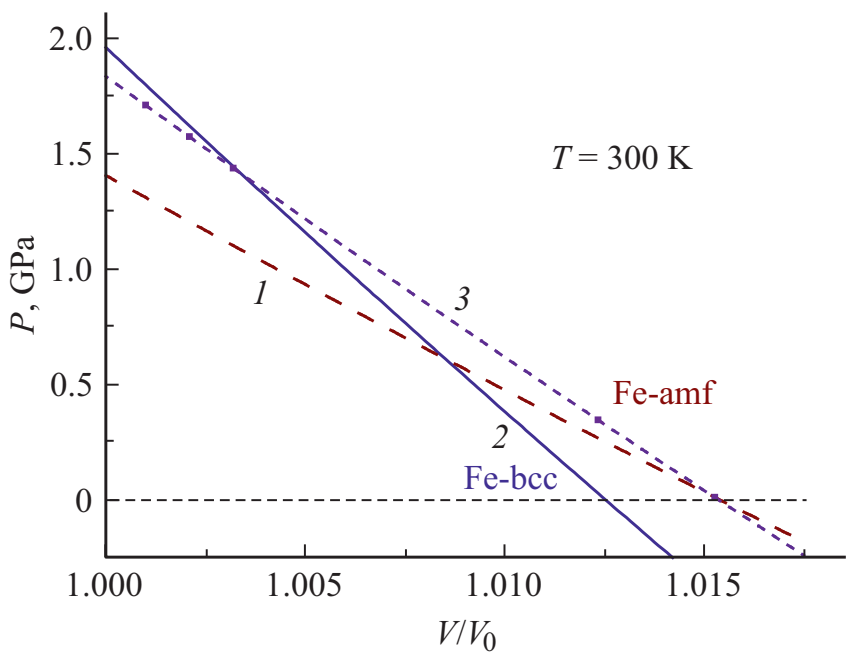

Рис. 3. Изотермы (при $300 \mathrm{~K})$ уравнения состояния (P в $[\mathrm{GPa}])$ железа с различной статической структурой. Штриховая линия 1 - расчет для точки 1 , сплошная линия $2-$ расчет для ОЦК-Fе, точечная линия $3-$ расчет для упаковки 3 из (4). 
и $P=0$, которые приводятся в литературе, лежат в следующих интервалах:

$\Theta(\mathrm{BCC}) /[\mathrm{K}]=420-476[11], 386-478[21]$,
$B_{T}(\mathrm{BCC}) /[\mathrm{GPa}]=164 \pm 7[22]-171.11[23]$,
$\alpha_{p}(\mathrm{BCC}) /\left[10^{-6} 1 / \mathrm{K}\right]=33-39[24]$,
$C_{p}(\mathrm{BCC}) /\left(N k_{\mathrm{B}}\right)=3.004[23], 3.002-3.022[21]$,
$\sigma(\mathrm{BCC}) /\left[10^{-3} \mathrm{~J} / \mathrm{m}^{2}\right]=1910-2525[25], 2280 \pm 43[26]$,
$\mu_{p}(\mathrm{BCC})=0.28[23]-0.32[21]$.
$\mathrm{B}[2], \quad$ исходя из значений скорости звука,

$\begin{array}{rrrr}\text { В } & {[2], \text { исходя из значений скорости звука, }} \\ \text { температуры Дебая аморфного } \mathrm{Fe} & \text { было }\end{array}$ получено: $\Theta($ Amorph $)=346.2 \mathrm{~K}$, а для жидкой фазы: $\Theta($ Liquid $)=364.7 \mathrm{~K}$.

На рис. 3-5 представлены изотермические (при $300 \mathrm{~K}$ ) зависимости различных термодинамических свойств для следующих статических структур: штриховая линия 1 - соответствует рыхлой аморфной упаковке (точка 1 на рис. $\left.2: k_{p}(1)=0.45556, k_{n}(1)=6.2793\right)$, сплошная линия 2 получена для ОЦК-Fe, точечная кривая 3 - соответствует плотной аморфной упаковке (точка 3: $k_{p}(3)=0.6237, k_{n}(3)=6.2793$ ).

На рис. 3 показана изотермическая (при $300 \mathrm{~K}$ ) зависимость от нормированного объема: $v / v_{o}=\left(c / r_{o}\right)^{3}=$ $=R^{-3}$, функции давления: $P=-\left(\partial f_{H} / \partial v\right)_{T}$ - для железа с различной структурой $(P$ в $[\mathrm{GPa}])$. Из рис. 3 и табл. 2 видно, что при $T=300 \mathrm{~K}$ и $P=0$ аморфные структуры имеет больший нормированный объем, причем значения $v / v_{o}$ в точках 1 и 3 почти совпадают. Но значения молярного объема $(V)$ в точках 1 и 3 при $T=300 \mathrm{~K}$ и $P=0$ существенно отличаются. Причем значение $V(3)$ лишь на $5.6 \%$ больше, чем $V($ ОЦК). Вывод о распухании вещества при аморфизации его структуры согласуется с результатами экспериментальных работ, указанных в обзорах [1-4,7,8].

На рис. 4 показаны изотермические (при $300 \mathrm{~K}$ ) зависимости от давления ( $P$ в $[\mathrm{GPa}])$ химического потенциала $(g-$ удельной энергии Гиббса), рассчитанной по

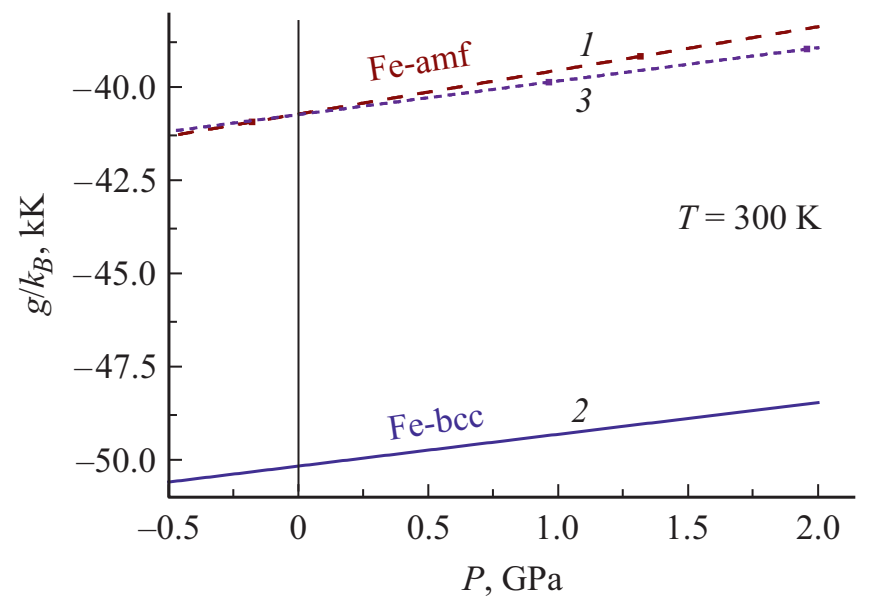

Рис. 4. Изотермические (при $300 \mathrm{~K}$ ) зависимости от давления химического потенциала $\left(g\right.$ в $\left.\left[10^{3} \mathrm{~K}\right]\right)$. Сплошная линия 2 - расчет для ОЦК-Fе, штриховая линия 1 - расчет для точки 1 , точечная линия 3 - расчет для точки 3 .

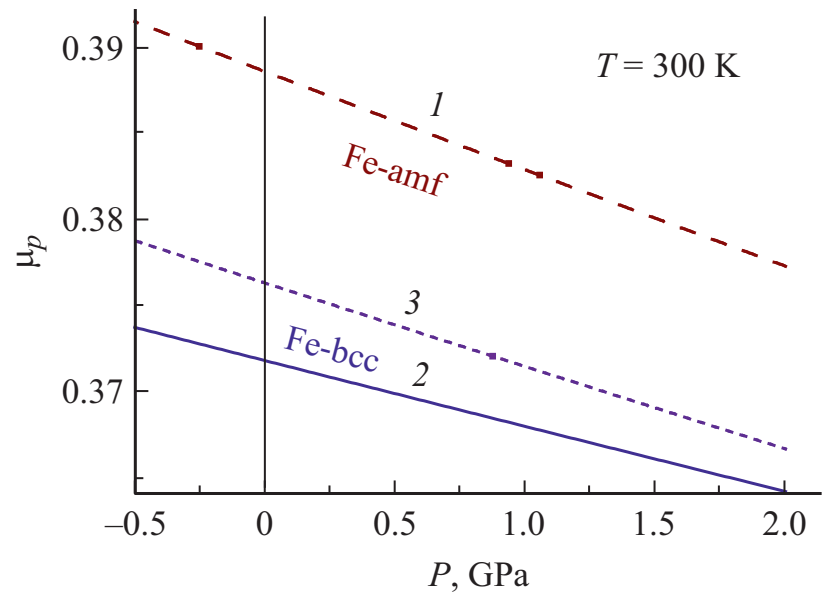

Рис. 5. Изотермические (при $300 \mathrm{~K}$ ) зависимости коэффициента Пуассона от давления для железа с различной статической структурой. Сплошная линия 2 - расчет для ОЦК-Fe, штриховая линия 1 - расчет для точки 1 , точечная линия 3 - расчет для точки 3 из (4).

формуле (13). Как видно из рис. 4 и табл. 2, химические потенциалы аморфных структур 1 и 3 при $T=300 \mathrm{~K}$ и $P=0$ совпадают. Это говорит о термодинамическом равновесии между состояниями в точках 1 и 3 при $T=300 \mathrm{~K}$ и $P=0$. Также видно, что при переходе из аморфного состояния в ОЦК структуру величина $g$ уменьшается, т.е. энергия при таком переходе выделяется.

На рис. 5 показаны изотермические (при $300 \mathrm{~K}$ ) зависимости от давления ( $P$ в $[\mathrm{GPa}])$ коэффициента Пуассона, рассчитанного по формуле (18). Как видно из рис. 5 и табл. 2 , величина $\mu_{p}$ при $T=300 \mathrm{~K}$ и $P=0$ для аморфных структур всегда выше, чем для кристалла. При этом значение $\mu_{p}(3)$ лишь на $1.1 \%$ больше, чем $\mu_{p}$ (ОЦК).

Из представленных в табл. 2 значений удельной поверхностной энергии следует, что величина $\sigma$ для аморфных структур всегда меньше, чем у кристалла. Поверхностная энергия твердого аморфного железа на $22 \%$ меньше чем у ОЦК железа, и на $11-16 \%$ больше, чем у жидкой фазы. Это должно приводить к аморфизации поверхности кристаллического металла. Этим же можно объяснить также появление „квази-жидкого“ слоя на поверхности кристалла [27,28].

Из представленных в табл. 2 значений удельной энтропии для четырех статических структур следует, что энтропия аморфных состояний всегда выше, чем энтропия кристалла. При этом наиболее разупорядоченной является структура в точке 2, которая, согласно данным из $[10]$, наиболее близка к области фазового перехода кристалл-жидкость:

$$
\begin{aligned}
k_{\text {psolidus }} & =0.494 \pm 0.002 \leq k_{p}(\text { Melting }) \\
& \leq k_{\text {pliquidus }}=0.545 \pm 0.002
\end{aligned}
$$


Отметим также, что структура в точке 2 близка к области, где наблюдается переход жидкости в стекло: $k_{p \text { Glass }}=0.58[29,30]$.

\section{Заключение}

Показано, что структура в точке 1 из (4) соответствует устойчивой статической упаковке жидкой аморфной фазы. Структура в точке 2 разделяет метастабильные аморфные статические структуры жидкой и твердой фаз. Структура в точке 3 соответствует неустойчивой статической твердой аморфной структуре, которая находится в термодинамическом равновесии с состоянием из точки 1. Из точки 3 система может флуктуационным путем либо перейти без изменения энергии в точку 1 , либо перейти с выделением энергии в кристаллическое состояние.

Определены значения параметров парного межатомного потенциала Ми-Леннард-Джонса для различных структур железа. Используя полученные параметры потенциала, изучено уравнение состояния железа при $T=300 \mathrm{~K}$ для различных статических структур. Рассчитаны термодинамические свойства различных статических структур железа при $T=300 \mathrm{~K}$ и $P=0$.

Впервые рассчитана удельная поверхностная энергия аморфного железа при $T=300 \mathrm{~K}$ и $P=0$. Показано, что поверхностная энергия твердого аморфного железа на $22 \%$ меньше, чем у ОЦК кристалла, и на $11-16 \%$ больше, чем у жидкой фазы. Это должно приводить к аморфизации поверхности кристаллического металла и появлению „квази-жидкого“ слоя как на поверхности кристалла, так и на поверхности твердой аморфной структуры.

\section{Благодарности}

Автор выражает благодарность С.П. Крамынину, Н.Ш. Газановой, 3.М. Сурхаевой и М.М. Гаджиевой за плодотворные дискуссии и помощь в работе.

\section{Финансирование работы}

Работа выполнена при финансовой поддержке РФФИ в рамках научного проекта № 18-29-11013_мк и Программы Президиума РАН № 6 (грант № 2-13).

\section{Конфликт интересов}

Авторы заявляют, что у них нет конфликта интересов.

\section{Список литературы}

[1] Белащенко Д.К. Компьютерное моделирование жидких и аморфных веществ. М.: МИСИС, 2005. 408 с.

[2] Singh R.N., Ali I. // Intern. J. Appl. Phys. Mathem. 2013. Vol. 3. N 4. P. 275-279. DOI: 10.7763/IJAPM.2013.V3.220
[3] Melnikov G., Emelyanov S., Ignatenko N., Manzhos O. // Key Eng. Mater. Trans Tech. Public. 2018. Vol. 781. P. 137-142. DOI: 10.4028/www.scientific.net/KEM.781.137

[4] Baule A., Morone F., Herrmann H.J., Makse H.A. // Rev. Modern Phys. 2018. Vol. 90. N 1. P. 015006 (1-56). DOI:10.1103/RevModPhys.90.015006

[5] Магомедов М.Н. // Журн. структурной химии. 2008. Т. 49. N 1. C. 164-167. [Magomedov M.N. // J. Structur. Chemi. 2008. Vol. 49. N 1. P. 166-159.] DOI: $10.1007 / \mathrm{s} 10947-008-0021-8$

[6] Kalikmanov V.I. // J. Chem. Phys. 2006. Vol. 124. N 12. P. 124505 (1-10). DOI: 10.1063/1.2178812

[7] Song C., Wang P., Makse H.A. // Nature. 2008. Vol. 453. N 7195. P. 629-632. DOI: $10.1038 /$ nature06981

[8] Hanifpour M., Francois N., Robins V., Kingston A., Allaei S.V., Saadatfar M. // Phys. Rev. E. 2015. Vol. 91. N 6. P. 062202 (1-9). DOI: 10.1103/PhysRevE.91.062202

[9] Магомедов М.Н. // Письма в ЖТФ. 2019. Т. 45. Вып. 20. C. 32-35. DOI: 10.21883/JTF.2020.10.49806.62-20

[Magomedov M.N. // Tech. Phys. Lett. 2019. Vol. 45. N 10. P. 1042-1046. DOI: 10.1134/S1063785019100249]

[10] Hoover W.G., Ree F.H. // J. Chem. Phys. 1968. Vol. 49. N 8. P. 3609-3617. DOI: 10.1063/1.1670641

[11] Магомедов М.Н. Изучение межатомного взаимодействия, образования вакансий и самодиффузии в кристаллах. M.: Физматлит, 2010. 544 с. [Magomedov M.N. Study of Interatomic Interaction, Vacancy Formation and SelfDiffusion in Crystals. M.: Fizmatlit, 2010.]

[12] Магомедов М.Н. // ЖТФ. 2013. Т. 83. Вып. 9. С. 56-62. [Magomedov M.N. // Tech. Phys. 2013. Vol. 58. N 9. P. 1297-1303.] DOI: $10.1134 / \mathrm{S} 106378421309020 \mathrm{X}$

[13] Жирибалько Л. Статистическая физика твердого тела. М.: Мир, 1975. 383 c. [Girifalco L.A. Statistical Physics of Materials. NY:: J. Wiley and Sons Ltd., 1973.]

[14] Магомедов М.Н. // ЖТФ. 2015. Т. 85. Вып. 11. С. 48-54. [Magomedov M.N. // Tech. Phys. 2015. Vol. 60. N 11. P. 1619-1625.] DOI: 10.1134/S1063784215110195

[15] Магомедов М.Н. // Кристаллография. 2017. Т. 62. № 3. C. 487-504. [Magomedov M.N. // Crystallography Reports. 2017. Vol. 62. N 3. P. 480-496.]

DOI: $10.1134 / \mathrm{S} 1063774517030142$

[16] Пичугин Е.Ф. // Известия вузов. Физика. 1962. № 6. C. 77-84. [Pichugin E.F. // Izvestiya Vysshikh Uchebnykh Zavedenii: Fizika. 1962. № 6. P. 77-84.]

[17] Магомедов М.Н. // ЖТФ. 2017. Т. 87. Вып. 4. С. 549-556. [Magomedov M.N. // Tech. Phys. 2017. Vol. 62. N 4. P. 569-576.] DOI:10.1134/S1063784217040156

[18] Huang X., Li F., Zhou Q., Meng Y., Litasov K.D., Wang X., Liu B., Cui T. // Scientific Reports. 2017. Vol. 6. P. 19923 (1-3). DOI: 10.1038/srep19923

[19] Batsanov S.S., Batsanov A.S. Introduction to structural chemistry. Heidelberg, London, NY: Springer Science \& Business Media, 2012. 545 p. DOI: $10.1007 / 978-94-007-4771-5$

[20] Luo Y.R. Bond dissociation energies / in CRC Handbook of Chemistry and Physics, 90th Edition, (Edited by David R. Lide). Boca Raton, FL: CRC Press (Taylor and Francis Group), 2009. 2804 p. https://notendur.hi.is/agust/rannsoknir/ papers/2010-91-CRC-BDEs-Tables.pdf 
[21] Зиновьев B.E. Теплофизические свойства металлов при высоких температурах. М.: Металлургия, 1989. 382 с. [Zinov'ev V.E. Teplofizicheskie svoistva metallov pri vysokikh temperaturakh (The Thermophysical Proper ties of Metals at High Temperatures), Moscow: Metallurgiya, 1989.]

[22] Wilburn D.R., Bassett W.A. // American Mineralogist. 1978. Vol. 63. N 5-6. P. 591-596. https://pubs.geoscienceworld.org/ $\mathrm{msa} / \mathrm{ammin} /$ article-abstract/63/5-6/591/40926

[23] Физические величины: Справочник / Под ред. И.С. Григорьева, E.3. Мейлихова. М.: Энергоатомиздат, 1991. 1232 с. [Handbook of Physical Quantities, Ed. by I.S. Grigoriev and E.Z. Meilikhov (Energoatomizdat, Moscow, 1991; CRC Press, Boca Raton, Florida, 1996)].

[24] Новикова С.И. Тепловое расширение твердых тел. М.: Наука, 1974. 294 с. [Novikova S.I., Thermal Expansion of Solids. M.: Nauka, 1974. 294 p.]

[25] Kumikov V.K., Khokonov Kh.B. // J. Appl. Phys. 1983. Vol. 54. N 3. P. $1346-1350$. DOI: $10.1063 / 1.332209$

[26] Kumikov V.K. // Mater. Sci. Eng. 1983. Vol. 60. N 3. P. L23-L24. DOI: 10.1016/0025-5416(83)90016-2

[27] Li Y., Somorjai G.A. // J. Phys. Chem. C. 2007. Vol. 111. N 27. P. 9631-9637. DOI: 10.1021/jp071102f

[28] Бокарев В.П., Горнев Е.С., Тодуа П.А. // Кристаллография. 2013. T. 58. № 1. C. 155-155.

DOI: $10.7868 / \mathrm{S} 0023476113010062$

[29] Pusey P.N., van Megen W. // Nature. 1986. V. 320. N 6060. P. 340-342. DOI: $10.1038 / 320340 \mathrm{a} 0$

[30] Campo M., Speck T. // J. Chem. Phys. 2020. V. 152. N 1. P. 014501. DOI: 10.1063/1.5134842 